\title{
Expression and activation of P38 MAP kinase in invasive ductal breast cancers: Correlation with expression of the estrogen receptor, HER2 and downstream signaling phosphorylated proteins
}

\author{
JEAN-LOUIS MERLIN ${ }^{1,2,3}$, ALEXANDRE HARLÉ ${ }^{1,2,3}$, MAËVA LION ${ }^{1,2,3}$, \\ CAROLE RAMACCI ${ }^{1}$ and AGNÈS LEROUX ${ }^{1,3}$ \\ ${ }^{1}$ Department of Biopathology, Institut de Cancérologie de Lorraine, 54519 Vandoeuvre les Nancy; \\ ${ }^{2}$ Université de Lorraine, Faculty of Pharmacy, 54001 Nancy; \\ ${ }^{3}$ CNRS UMR 7039 CRAN, 54506 Vandoeuvre les Nancy, France
}

Received May 29, 2013; Accepted June 28, 2013

DOI: $10.3892 /$ or.2013.2645

\begin{abstract}
MAP kinase signaling proteins have major implications in the molecular oncogenesis of breast cancers and have been extensively investigated as putative targets for therapy. This study reports the investigation of the expression of P38 MAPK and its phosphorylated form (p-P38 MAPK) in clinical specimens of invasive breast carcinomas and their correlation with estrogen receptor (ER) and HER2 expression, as well as MAPK and PI3 kinase-AKT pathway signaling phosphorylated proteins. Expression levels of P38 MAPK and p-P38 MAPK as well as p-AKT, p-GSK3 $\beta$, p-S6 kinase, $\mathrm{p}-\mathrm{MEK} 1$ and p-ERK1/2 were quantitatively assessed using multiplex bead immunoassay in frozen specimens from 45 invasive ductal breast cancers. Twenty-nine specimens were $\mathrm{ER}^{+}, 15$ were $\mathrm{HER} 2^{+}$and 10 were triple-negative breast cancers (TNBCs). P38 MAPK was found to be expressed in all tumor specimens and was significantly $(\mathrm{P}=0.002)$ overexpressed in $\mathrm{ER}^{+}$tumors. P38 MAPK expression was lower in TNBCs than in all of the other tumors. The median expression of p-P38 MAPK was also higher in $\mathrm{ER}^{+}$tumors while lower in the TNBCs. HER2 status had no effect on P38 MAPK and p-P38 MAPK expression. No variation in the phosphorylation rate of P38 MAPK was observed in relation with ER, HER2 or TNBC status. Significantly higher $(\mathrm{P}=0.0048)$ expression of p-AKT was observed in HER $2^{+}$tumors. No significant difference in $\mathrm{p}-\mathrm{MEK} 1, \mathrm{p}-\mathrm{GSK} 3 \beta$ and $\mathrm{p}-\mathrm{S} 6 \mathrm{~K}$ expression was found in any other comparisons based on ER and HER2 expression
\end{abstract}

Correspondence to: Professor Jean-Louis Merlin, Institut de Cancérologie de Lorraine, 6 Avenue de Bourgogne, CS30519, 54519 Vandoeuvre les Nancy, France

E-mail: jl.merlin@nancy.unicancer.fr

Key words: breast cancer, signaling, MAPK, estrogen receptor, HER2 subtypes. Investigation of the expression of multiple phosphorylated signaling proteins can be used for personalized targeted therapy. In invasive breast cancer, the overexpression of P38 MAPK may serve as a biomarker for the evaluation of P38 MAPK inhibitors.

\section{Introduction}

Breast cancer is the most common cancer in women with more than one million new cases. Breast cancer is one of the leading causes of cancer-related mortality in women (1). Apart from surgery, adjuvant radiation therapy and chemotherapy, treatment of breast cancer is based on the identification of molecular targets, mainly estrogen receptor (ER) expression, HER 2 overexpression and amplification. In ER-expressing tumors, endocrine therapy consisting of mainly tamoxifen and anti-aromatase drugs is prescribed. In human epidermal growth factor receptor 2 (HER2)-positive (HER2 ${ }^{+}$) tumors, anti-HER2-targeted therapies such as the anti-HER2 monoclonal antibody trastuzumab and the tyrosine kinase inhibitor lapatinib have been proposed. Moreover, in HER2 ${ }^{+}$tumors also expressing ER, the combination of both endocrine and anti-HER 2 therapies have been proven to improve the outcome of patients through the blockade of signaling crosstalk leading to resistance to ER-targeted therapy. In triple-negative breast cancers (TNBCs) i.e. ER- $\mathrm{PR}^{-}$and HER2- tumors, no targeted therapy has been proven effective to date, and the molecular characteristics of TNBCs have been extensively studied in order to identify putative molecular targets for drug development. Since several different growth factor pathways can stimulate breast cell growth, targeting a unique pathway may have limited effect on the inhibition of breast cancer proliferation, and the inhibition of signal transduction at a deeper point in the cascade has been envisaged.

In this context, mammalian target of rapamycin (mTOR) has been identified as the point of convergence of many mitogenic signals, which has led to the recent registration of the mTOR inhibitor everolimus in association with anti- 
aromatase therapy for $\mathrm{ER}^{+}$breast cancers (2). Another point of convergence of intracellular downstream growth factor receptor signaling is the $\mathrm{P} 38$ mitogen-activated protein kinase family (P38 MAPK). P38 MAPK is a member of the MAPK family which includes the extracellular signal-regulated kinase (ERK), the c-Jun N-terminal kinase (JNK), and P38 MAPK (3). P38 MAPK is comprised of four isoforms $(\alpha$, $\beta, \gamma$ and $\delta$ ) that can be activated by various growth factors, inflammatory cytokines, and chemical or physical stress. The $\alpha$ isoform is the most abundant and is subject to a larger inter-individual variability than the other three isoforms (12). P38 MAPK plays a complex role in the regulation of cell growth, differentiation, apoptosis, and responses to inflammation or stress $(4,5)$. P38 MAPK activity was found to be upregulated in breast, head and neck carcinomas, lymphomas, gliomas and squamous cell carcinomas (6).

In breast cancer, a high level of expression of P38 MAPK has been found to correlate with poor prognosis and to be involved in invasiveness and metastasis in relation with the urokinase plasminogen activator system (7). Activation of P38 MAPK has been observed in Scarff-Bloom-Richardson (SBR) grade 2 or 3 ductal tumors (8). Expression of phosphorylated-P38 MAPK (p-P38MAPK) has been reported in $\sim 20 \%$ of primary breast carcinomas (9). P38 MAPK overexpression has been correlated with HER 2 amplification and tamoxifen resistance (10) and has been proposed as a potential prognostic marker in breast cancer (11). Specifically, phosphorylation of P38 MAPK was found to be a negative prognostic indicator in HER2-negative, lymph node-positive breast cancers (9).

The role of P38 MAPK in the regulation of breast cancer cell proliferation remains to be elucidated and has been suggested to have dual activities that include regulation of survival and proliferation depending on the expression of mutant TP53 (12) as observed in most ER- breast tumors therefore justifying the development of P38 MAPK inhibitors for the treatment of TP53-mutated, ER ${ }^{-}$breast cancers or TNBCs. Furthermore, the activation of P38 MAPK has recently been reported to regulate signaling by EGFR/c-Src crosstalk in breast cancer (13).

In addition, P38 MAPK has been recently reported to play a role in the resistance of $\mathrm{ER}^{+}$breast tumors to endocrine therapy (14). Although the cellular mechanisms underlying the development of tamoxifen resistance in breast cancer cells are not totally understood, recent research has found that alteration of the signaling pathways (15-17) can decrease the cell sensitivity to tamoxifen. More precisely, the development of crosstalk between ER and growth factor-mediated activation of the MAPK cascade, through the activation of HER2 has been reported to increase both genomic and non-genomic ER actions in breast cancer leading to tamoxifen resistance. This justifies the combination of endocrine therapy together with aromatase inhibitors and anti-HER2 therapies with trastuzumab-based or lapatinib-based therapies for breast cancer (18). Recent studies have noted a positive correlation between activated P38 MAPK levels and tamoxifen resistance (19). P38 MAPK has been reported to potentiate ER agonist activity through increased phosphorylation of ER and enhanced ER signaling through coactivator regulation (20). P38 MAPK has been shown to play a role in breast cancer progression and inva- sion (21) in association with other signaling proteins such as integrins and urokinase plasminogen activator (22) as well as H-RAS (23).

P38 MAPK isoform $\gamma$ has been recently shown to be selectively activated by exposure to tamoxifen, consequently recruiting nonclassical ER signaling and increased estrogen cell sensitivity (24). Therefore, increased P38 MAPK activation could define a more malignant, resistant and metastatic breast cancer phenotype and justify the evaluation of P38 MAPK inhibitors in the treatment of invasive and tamoxifenresistant breast carcinomas (14). A number of P38 MAPK inhibitors are currently being investigated in clinical trials (25).

In the present study, we investigated the expression of P38 MAPK and p-P38 MAPK in clinical specimens of invasive breast carcinomas. We first investigated the correlation of their expression with ER and HER2 expression, and subsequently evaluated the correlation with expression levels of MAPK and PI3K signaling phosphorylated proteins such as p-AKT, p-GSK3 $\beta$, p-S6 kinase, p-MEK1 and p-ERK1/2 quantitatively determined using multiplex bead immunoassay as previously described and validated in breast cancer (26).

\section{Materials and methods}

Patients and tumor characteristics. Frozen tumor samples of breast cancer from 45 patients with infiltrative ductal carcinoma were obtained from the tumor bank of our Institute (agreement with French National Cancer Institute and Ministry of Health). All patients were informed of the tumor banking procedure and no opposition was expressed. The median age at diagnosis was 56.3 years (range, 28-91).

Breast cancer tissues macroscopically selected by the pathologists were obtained immediately after surgery and were shock frozen in liquid nitrogen then cryopreserved at $-80^{\circ} \mathrm{C}$. The mean tumor (SD) specimen weight was 15.2 (4.2) $\mathrm{mg}$. None of the patients received any preoperative adjuvant endocrine therapy or chemotherapy. Thirty-four tumors were SBR grade 3 (Scarff-Bloom-Richardson) and 11 tumors were grade 2 .

Immunohistochemistry (BenchMark Ventana) was used to detect estrogen and progesterone receptor expression and HER-2 overexpression as part of the routine clinical diagnostics using polyclonal antibody A485 (Dako, Trappes, France) immunostaining of HER2 and monoclonal antibodies 6F11 and Pgr312 (both from Novocastra, Leica Microsystèmes, Nanterre, France) for determination of estrogen and progesterone expression, respectively.

Protein extraction. The tumor specimens were first disrupted using steel bead TissueLyser (Qiagen, Courtaboeuf, France) for $15 \mathrm{~min}$, and then exposed to the lysis solution (Cell Lysis kit, Bio-Rad, Marnes-la-Coquette, France) containing PMSF anti-protease for $10 \mathrm{sec}$. After centrifugation $(4,500 \mathrm{x} \mathrm{g}$ for $20 \mathrm{~min}$ at $4^{\circ} \mathrm{C}$ ), the protein-containing supernatants were collected and stored frozen at $-80^{\circ} \mathrm{C}$ until analysis. Before being analyzed, the protein concentration was determined in each extract using $690 \mathrm{~nm}$ colorimetric DC protein assay kit (Bio-Rad) based on Lowry technique and adjusted to $250 \mu \mathrm{g} / \mathrm{ml}$. 
Multiplex bead immunoassay. The expression of the signaling phosphoproteins was analyzed using multiplex bead immunoassay as described and validated previously (26). Briefly, protein extracts were transferred into 96-well dishes and diluted with $25 \mu \mathrm{l}$ buffered solution. Fluorescence capturing beads coupled to antibodies directed against P38 MAPK, p-P38 MAPK, p-AKT, p-GSK3 $\beta$, p-P70S6K, p-MEK1, p-ERK1/2 phosphoproteins were mixed. The beads were added into each well and incubated overnight at $37^{\circ} \mathrm{C}$. Biotinylated antibodies and then streptavidin-phycoerythrin solution were then added. The positive control consisting of standard protein extract from cell lines was added to each series. The multiwell plates were then analyzed according to the manufacturer's instructions (Bio-Plex; Bio-Rad, Hercules, CA, USA). Frozen protein extracts from an EGFR-overexpressing human breast cancer cell line exposed to EGF were used as positive controls as reported previously (26). The results were recorded as mean fluorescence intensities expressed as arbitrary units and considered as significant when exceeding a signal/noise ratio of 3 .

Statistics. All analyses were performed as triplicate and are presented as mean fluorescence intensities (SD). All statistical analyses were performed using the Wilcoxon test using R software (v.2.15.1.; the R Foundation for Statistical Computing) and the level of significance was set at $\mathrm{P}<0.05$.

\section{Results}

Immunohistochemistry. The breat cancer tumor characteristics are summarized in Table I. The ER status as determined by immunohistochemistry was positive in 29 patients $(64 \%)$ and negative in 16 patients (36\%). Progesterone receptor (PR) status was positive in 18 patients $(40 \%)$ and negative in 27 patients $(60 \%)$. Fifteen tumors $(33 \%)$ were HER2 $2^{+}$and 30 tumors $(67 \%)$ were HER2-. Ten $(22 \%)$ tumors were triplenegative breast cancers (TNBCs), i.e. ER', $\mathrm{PR}^{-}$and HER2-

P38 MAPK and phosphorylated-P38 MAPK expression. P38 MAPK and p-P38 MAPK were found to be expressed in nearly all tumor specimens $(44 / 45,98 \%)$ and were significantly $(\mathrm{P}=0.0016)$ overexpressed in $\mathrm{ER}^{+}$tumors (Fig. 1A). The median expression of p-P38 MAPK was also higher in $\mathrm{ER}^{+}$when compared with that in ER- tumors. HER2 status had no influence on P38 MAPK and p-P38 MAPK expression (Fig. 1B). P38 MAPK expression was lower in TNBCs (Fig. 1C) when compared with the expression level in all other tumor types as was p-P38MAPK expression but without reaching statistical significance. No significant variation in the phosphorylation rate of P38 MAPK, as calculated from the phosphorylated/ unphosphorylated P38 MAPK expression ratios, was observed in association with ER, HER2 and TNBC status, or SBR grade.

Phosphorylated-AKT and phosphorylated-ERK1/2 expression. Significant expression of p-AKT and p-ERK1/2 was detected in 33/45 (73\%) and 17/45 (38\%) of the tumor extracts, respectively. p-AKT expression was found to be significantly higher $(\mathrm{P}=0.0048)$ in HER2 ${ }^{+}$tumors (Fig. 2) than in HER2 tumors. No other significant difference was observed regarding either ER and TNBC status or SBR grade. No difference in
Table I. Patient demographics and tumor characteristics.

\begin{tabular}{lr}
\hline Characteristics & Patients, $\mathrm{n}(\%)$ \\
\hline No. of patients & $45(100.0)$ \\
Age (years) & \\
$\leq 50$ & $13(28.9)$ \\
$51-69$ & $24(53.3)$ \\
$\geq 70$ & $8(17.8)$ \\
Tumor size (mm) & \\
T1 (10-20) & $16(35.6)$ \\
T2 (21-50) & $26(57.8)$ \\
T3 (>51) & $3(6.7)$ \\
SBR grade & \\
SBR 2 & $11(24.4)$ \\
SBR 3 & $34(75.6)$ \\
Hormone receptor status & \\
ER ${ }^{+}$ & $29(64.4)$ \\
ER & $16(35.6)$ \\
PR ${ }^{+}$ & $18(40.0)$ \\
PR & $27(60.0)$ \\
HER2 status & \\
Positive & $15(33.3)$ \\
Negative & $30(66.7)$ \\
Triple-negative & $10(22.2)$ \\
\hline
\end{tabular}

ER, estrogen receptor; PR, progesterone receptor; SBR, Scarff-BloomRichardson; HER2, human epidermal growth factor receptor 2.

p-ERK1/2 expression was observed regarding ER, HER2 and TNBC status. Expression of p-ERK1/2 was found to be significantly higher $(\mathrm{P}=0.0235)$ in $\mathrm{SBR}$ grade 3 than in $\mathrm{SBR}$ grade 2 tumors (Fig. 3 ).

Expression of other phosphorylated-signaling proteins $M E K 1$, GSK $3 \beta$, S6K. Significant expression of p-MEK1, p-GSK3 $\beta$ and p-S6K was detected in 39/45 (87\%), 31/45 (69\%) and $37 / 45(82 \%)$ of the protein extracts, respectively. No significant difference in $\mathrm{p}-\mathrm{MEK} 1, \mathrm{p}-\mathrm{GSK} 3 \beta$ and $\mathrm{p}-\mathrm{S} 6 \mathrm{~K}$ expression was evidenced regarding either ER and TNBC status or SBR grade (data not shown). No significant correlation was found between the expression levels of any of the phosphorylated proteins.

\section{Discussion}

In breast cancer, P38 MAPK expression has previously been correlated with invasiveness and poor prognosis (8-11).

In the present study, we compared the expression of P38 MAPK and p-P38 MAPK in clinical specimens of invasive breast carcinomas in association with ER, HER2 and SBR grade and aimed to ascertain a correlation between P38 MAPK expression or activation of MEK/ERK and the AKT/mTOR signaling pathways.

In our series, expression of P38 MAPK and p-P38 MAPK was observed in nearly all tumor specimens. This was consis- 

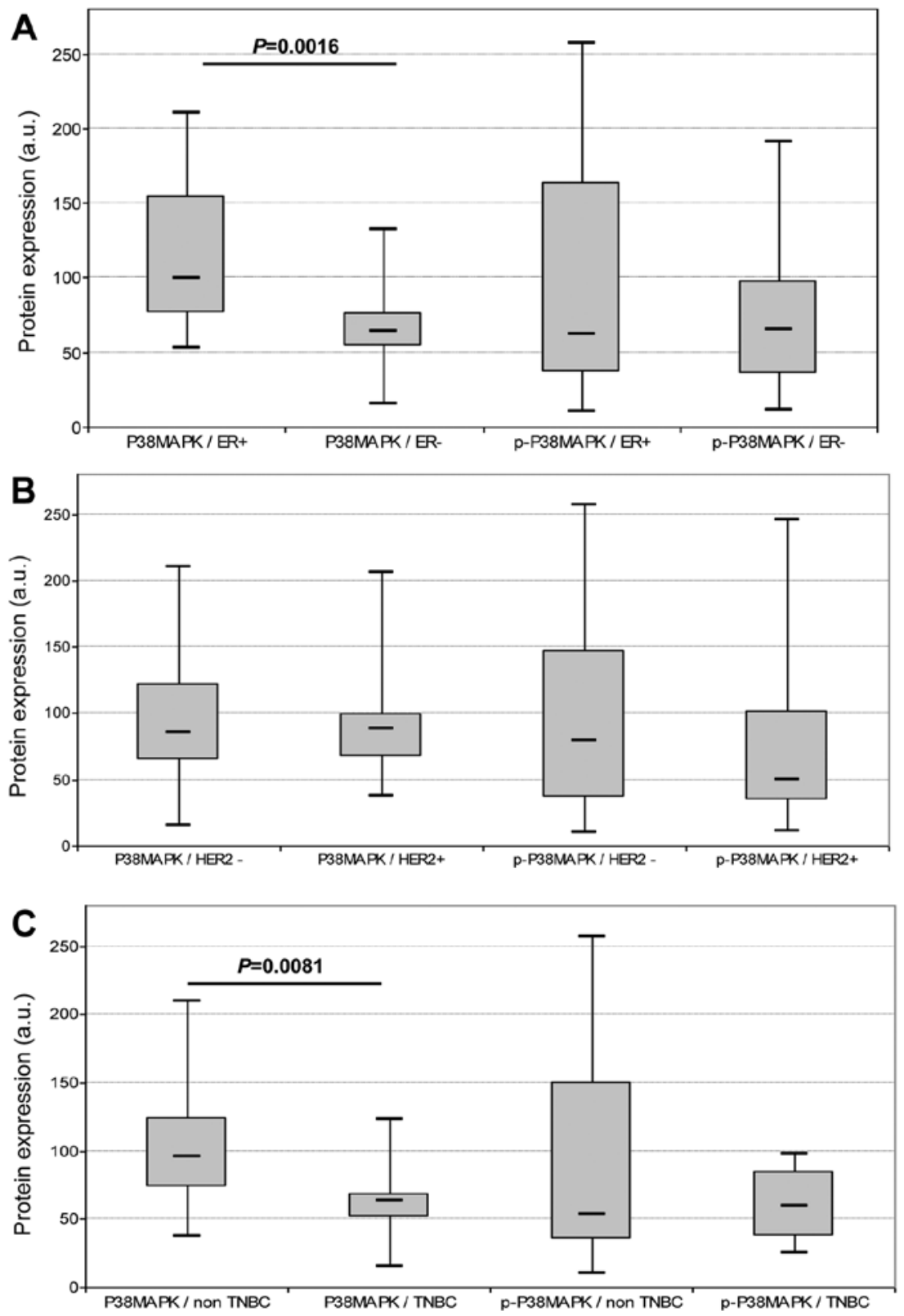

Figure 1. Expression of P38 MAPK and its phosphorylated form (p-P38 MAPK) in clinical specimens of invasive breast cancers. The results are presented as box-and-whisker plots, illustrating the median (central bar), 1st and 3rd quartiles (bottom and top of the grey box), minimum and maximum values (lower and upper bars). Expression in (A) estrogen-positive (ER ${ }^{+}$) and ER-negative (ER') tumors; (B) HER2-positive (HER2 ${ }^{+}$) and HER2-negative (HER2) tumors; (C) triple-negative breast cancer (TNBC) and non-TNBC.

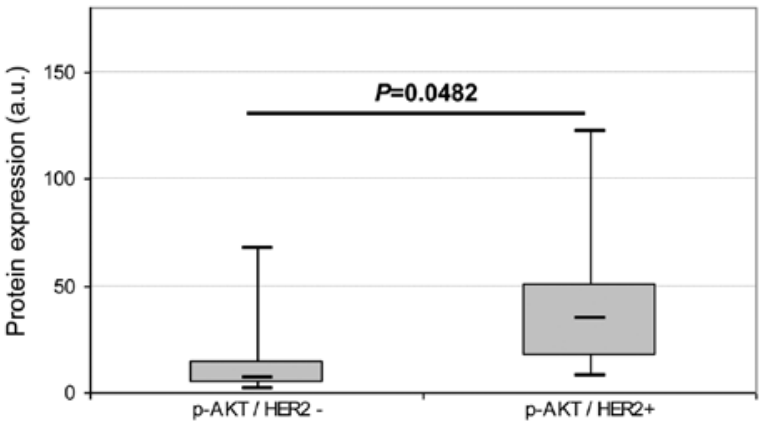

Figure 2. Expression of phosphorylated-AKT (p-AKT) in HER2-positive $\left(\mathrm{HER}^{+}\right)$and HER2-negative (HER2) tumors. The results are presented as box-and-whisker plots, illustrating the median (central bar), 1st and 3rd quartiles (bottom and top of the grey box), minimum and maximum values (lower and upper bars).

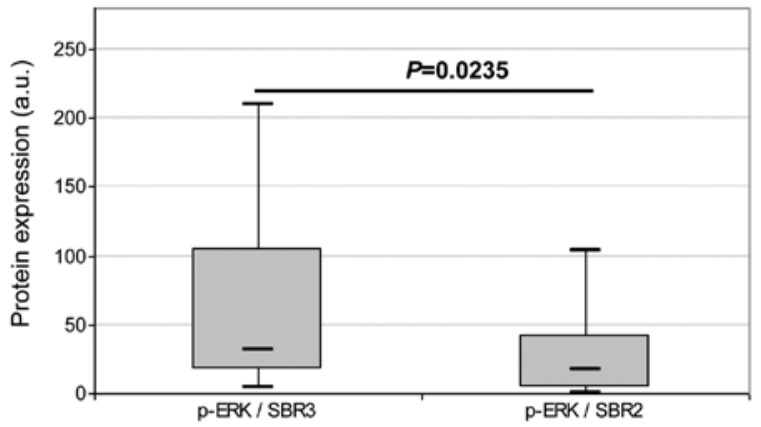

Figure 3. Expression of phosphorylated-ERK1/2 (p-ERK) in SBR grade 2 (SBR2) and grade 3 (SBR3) tumors. The results are presented as box-andwhisker plots, illustrating the median (central bar), 1st and 3rd quartiles (bottom and top of the grey box), minimum and maximum values (lower and upper bars). 
tent with previously published data (11) reporting P38 MAPK and p-P38 MAPK expression in 100 and 89\% of specimens, respectively, using western blot analysis, and $70 \%$ when IHC was used.

We report here that P38 MAPK was expressed at a higher level in $\mathrm{ER}^{+}$when compared with $\mathrm{ER}^{-}$tumors without post-transductional activation since no variation in the phosphorylation rate of P38 MAPK was evidenced. This is consistent with previously published data $(10,27)$ revealing the great interest in P38 MAPK in ER ${ }^{+}$tumors. P38 MAPK has been reported to be activated by anti-estrogens apart from ER their main target, resulting in a switch in ER signaling from its classical pathway, involving the estrogen response element (ERE) DNA domain, to the AP1-dependent non-classical pathways; therefore, activation of P38 MAPK can ultimately decrease the cellular response to endocrine therapy. Based on this concept, P38 MAPK has been proposed as a biomarker for resistance to endocrine therapy, and quantitative assessment of P38 MAPK expression and the detection of its activation in breast tumors may represent a new approach to predict the resistance of breast cancer to endocrine therapy. Furthermore, inhibition of P38 MAPK in ER tumors could restore ER expression and therefore restablish the sensivity to endocrine therapy (28).

Moreover, evaluation of the molecular pathway may even be proposed for specimens obtained at recurrence since the molecular pathways driving tumor growth could be altered along with tumor progression (10).

An incomplete understanding of the complex mechanisms exists concerning the relationship between MAPK activation and expression of hormone receptors and HER2 in breast carcinoma in vitro. In effusion specimens, p38 activation was reported to be inversely associated with the intensity of HER2 membrane expression (11).

In this context, although we did not observe any inverse relationship between HER2 and p-P38 MAPK expression, our results revealed that expression of P38 MAPK was significantly lower in TNBCs than in the other tumor subtypes. This may be reconsidered if a more specific approach of selective inhibition of P38 MAPK isoforms can be envisaged, as recent preclinical studies have demonstrated the important role played by the P38 MAPK $\gamma$ isoform in TNBCs in relation with its marked induction of cell cycle arrest in the $\mathrm{G}(2) / \mathrm{M}$ phase (29) and its effect on the cellular sensitivity to topoisomerase II inhibitors (30). Stimulation of topo II $\alpha$ gene expression by P38 MAPK $\gamma$ may contribute to increased topo II $\alpha$ levels and enhanced antitumor activity of topo II inhibitors (24), therefore opening the field for the investigation of selective inhibitors of the P38 MAPK $\gamma$ isoform in combination with chemotherapy.

p-ERK was detected in $73 \%$ of the tumor specimens, consistent with data reporting significant $\mathrm{p}$-ERK expression in 69 to $96 \%$ of breast tumors $(11,31,32)$. A low expression rate (35\%) was only reported in one cohort (33). In our study, the expression of $\mathrm{p}-\mathrm{ERK}$ was higher in high grade tumors (SBR3) consistent with data linking the activation of ERK with breast cancer cell proliferation (33).

p-AKT was detected in $38 \%$ of the tumor specimens and at a higher level in HER2 $2^{+}$tumors, consistent with data previously published using IHC which reported p-AKT cytoplasmic and nuclear expression rates of 36 and $29 \%$, respectively, in invasive ductal breast tumors and higher activation of AKT in HER2 ${ }^{+}$tumors (34). In this study (34), a correlation was observed between nuclear p-AKT and nuclear ER and PR expression while no difference was observed for cytoplasmic p-AKT expression and cytoplasmic ER and PR expression. This is consistent with our data showing an absence of a correlation between either ER or PR and p-AKT expression since when using total protein extract analysis no difference can be determined between cytoplasmic and nuclear compartments.

As a whole, we did not find any correlation between p-AKT, p-ERK expression and P38MAPK or p-P38MAPK expression either in the total population of this study or in ER, HER2 or TNBC subgroups. Similar findings have been reported and no significant correlations were evidenced between ER and levels of p-P38MAPK, p-AKT, or p-ERK $(10,11)$. Only several correlations have been reported between p-P38 MAPK and p-AKT and between p-P38 MAPK and p-ERK in a global population of tumor specimens from untreated patients analyzed using IHC (10). Similar to other research (11), we did not find any relationship between HER2 and p-ERK.

Collectively, our data indicate that the regulation of P38 MAPK was not directly linked to any of the investigated signaling pathways and could be considered as an independent biomarker in breast cancer. This also confirms the complexity of breast cancer oncogenesis, involving the recruitment of multiple signaling pathways.

In conclusion, P38 MAPK expression and activation are frequently observed in breast carcinoma and appear to be positively associated with the expression of the ER. Our results confirm the capability of breast cancer cells to activate P38 MAPK-mediated stress mechanisms and that P38 MAPK may represent a biological target for $\mathrm{ER}^{+}$breast cancer. The frequent concomitant activation of P38 MAPK, ERK and AKT indicates that breast tumor growth involves the activation of multiple signaling pathways, probably explaining the multiple mechanisms by which tumor cells develop resistance. Control of tumor growth should therefore entail the inhibition of all signaling pathways by combining multiple targeted therapies either in concomitant or in sequential use.

\section{Acknowledgements}

The present study was supported by the French 'Ligue contre le Cancer, Comité Inter-régional Grand Est' and Alexis Vautrin private research funds.

\section{References}

1. Jemal A, Bray F, Center MM, Ferlay J, Ward E and Forman D: Global cancer statistics. CA Cancer J Clin 61: 69-90, 2011.

2. Baselga J, Campone M, Piccart M, et al: Everolimus in postmenopausal hormone-receptor-positive advanced breast cancer. N Engl J Med 366: 520-529, 2012.

3. Maemura M, Iino Y, Koibuchi Y, Yokoe T and Morishita Y: Mitogen-activated protein kinase cascade in breast cancer. Oncology 57 (Suppl 2): 37-44, 1999.

4. Ono $\mathrm{K}$ and Han J: The p38 signal transduction pathway: activation and function. Cell Signal 12: 1-13, 2000.

5. Frigo DE, Basu A, Nierth-Simpson EN, et al: p38 mitogenactivated protein kinase stimulates estrogen-mediated transcription and proliferation through the phosphorylation and potentiation of the p160 coactivator glucocorticoid receptorinteracting protein 1. Mol Endocrinol 20: 971-983, 2006. 
6. Wagner EF and Nebreda A: Signal integration by JNK and p38 MAPK pathways in cancer development. Nat Rev Cancer 9: 537-549, 2009.

7. Tang L and Han X: The urokinase plasminogen activator system in breast cancer invasion and metastasis. Biomed Pharmacother 67: 179-182, 2013

8. Salh B, Marotta A, Wagey R, Sayed M and Pelech S: Dysregulation of phosphatidylinositol 3-kinase and downstream effectors in human breast cancer. Int J Cancer 98: 148-154, 2002

9. Esteva FJ, Sahin AA, Smith TL, et al: Prognostic significance of phosphorylated P38 mitogen-activated protein kinase and HER-2 expression in lymph node-positive breast carcinoma. Cancer 100: 499-506, 2004

10. Gutierrez MC, Detre S, Johnston S, et al: Molecular changes in tamoxifen-resistant breast cancer: relationship between estrogen receptor, HER-2, and p38 mitogen-activated protein kinase. J Clin Oncol 23: 2469-2476, 2005.

11. Davidson B, Konstantinovsky S, Kleinberg L, et al: The mitogenactivated protein kinases (MAPK) p38 and JNK are markers of tumor progression in breast carcinoma. Gynecol Oncol 102: 453-461, 2006

12. Chen L, Mayer JA, Krisko TI, et al: Inhibition of the p38 kinase suppresses the proliferation of human ER-negative breast cancer cells. Cancer Res 69: 8853-8861, 2009.

13. Mueller KL, Powell K, Madden JM, Eblen ST and Boerner JL: EGFR tyrosine 845 phosphorylation-dependent proliferation and transformation of breast cancer cells require activation of p38 MAPK. Transl Oncol 5: 327-334, 2012.

14. Antoon JW, Bratton MR, Guillot LM, et al: Pharmacology and anti-tumor activity of RWJ67657, a novel inhibitor of p38 mitogen activated protein kinase. Am J Cancer Res 2: 446-458, 2012.

15. Ghayad SE, Vendrell JA, Ben Larbi S, Dumontet C, Bieche I and Cohen PA: Endocrine resistance associated with activated ErbB system in breast cancer cells is reversed by inhibiting MAPK or PI3K/Akt signaling pathways. Int J Cancer 126: $545-562,2010$

16. Normanno N, Di Maio M, De Maio E, et al: Mechanisms of endocrine resistance and novel therapeutic strategies in breast cancer. Endocr Relat Cancer 12: 721-747, 2005.

17. Musgrove EA and Sutherland RL: Biological determinants of endocrine resistance in breast cancer. Nat Rev Cancer 9: 631-643, 2009.

18. Cortés J, Saura C, Bellet M, et al: HER2 and hormone receptorpositive breast cancer - blocking the right target. Nat Rev Clin Oncol 8: 307-311, 2011

19. Massarweh S, Osborne CK, Creighton CJ, et al: Tamoxifen resistance in breast tumors is driven by growth factor receptor signaling with repression of classic estrogen receptor genomic function. Cancer Res 68: 826-833, 2008.

20. Lee $\mathrm{H}$ and Bai W: Regulation of estrogen receptor nuclear export by ligand-induced and p38-mediated receptor phosphorylation. Mol Cell Biol 22: 5835-5845, 2002.

21. Reddy KB, Nabha SM and Atanaskova N: Role of MAP kinase in tumor progression and invasion. Cancer Metastasis Rev 22: $395-403,2003$
22. Chen J, Baskerville C, Han Q, Pan ZK and Huang S: $\alpha(v)$ integrin, p38 mitogen-activated protein kinase, and urokinase plasminogen activator are functionally linked in invasive breast cancer cells. J Biol Chem 276: 47901-47905, 2001.

23. Kim MS, Lee EJ, Kim HR and Moon A: p38 kinase is a key signaling molecule for $\mathrm{H}$-Ras-induced cell motility and invasive phenotype in human breast epithelial cells. Cancer Res 63: 5454-5461, 2003.

24. Qi X, Zhi H, Lepp A, et al: p38 $\gamma$ mitogen-activated protein kinase (MAPK) confers breast cancer hormone sensitivity by switching estrogen receptor (ER) signaling from classical to nonclassical pathway via stimulating ER phosphorylation and c-Jun transcription. J Biol Chem 287: 14681-14691, 2012.

25. Banerjee A, Koziol-White C and Panettieri R Jr: p38 MAPK inhibitors, IKK2 inhibitors, and TNFa inhibitors in COPD. Curr Opin Pharmacol 12: 287-292, 2012.

26. Chergui F, Chrétien AS, Bouali S, et al: Validation of a phosphoprotein array assay for characterization of human tyrosine kinase receptor downstream signaling in breast cancer. Clin Chem 55: 1327-1336, 2009

27. Svensson S, Jirström K, Rydén L, et al: ERK phosphorylation is linked to VEGFR2 expression and Ets-2 phosphorylation in breast cancer and is associated with tamoxifen treatment resistance and small tumours with good prognosis. Oncogene 24: 4370-4379, 2005

28. Bhatt S, Xiao Z, Meng Z and Katzenellenbogen BS: Phosphorylation by $\mathrm{p} 38$ mitogen-activated protein kinase promotes estrogen receptor $\alpha$ turnover and functional activity via the SCF(Skp2) proteasomal complex. Mol Cell Biol 32: 1928-1943, 2012.

29. Meng F, Zhang H, Liu G, et al: p38 $\gamma$ mitogen-activated protein kinase contributes to oncogenic properties maintenance and resistance to poly (ADP-ribose)-polymerase-1 inhibition in breast cancer. Neoplasia 13: 472-482, 2011

30. Qi X, Hou S, Lepp A, et al: Phosphorylation and stabilization of topoisomerase II $\alpha$ protein by p38 $\gamma$ mitogen-activated protein kinase sensitize breast cancer cells to its poison. J Biol Chem 286: 35883-35890, 2011.

31. Milde-Langosch K, Bamberger AM, Rieck G, et al: Expression and prognostic relevance of activated extracellular-regulated kinases (ERK1/2) in breast cancer. Br J Cancer 92: 2206-2215, 2005.

32. Linderholm BK, Hellborg H, Johansson U, Skoog L and Lehtiö J: Vascular endothelial growth factor receptor 2 and downstream p38 mitogen-activated protein kinase are possible candidate markers of intrinsic resistance to adjuvant endocrine treatment in steroid receptor-positive breast cancer. Breast Cancer Res Treat 125: 457-465, 2011.

33. Hermanto U, Zong CS and Wang LH: Inhibition of mitogenactivated protein kinase kinase selectively inhibits cell proliferation in human breast cancer cells displaying enhanced insulin-like growth factor I-mediated mitogen-activated protein kinase activation. Cell Growth Differ 11: 655-664, 2000.

34. Park SS and Kim SW: Activated Akt signaling pathway in invasive ductal carcinoma of the breast: Correlation with HER2 overexpression. Oncol Rep 18: 139-143, 2007. 